\title{
GLOBAL VLBI OBSERVATIONS OF THE CENTRAL REGION IN NGC 3079
}

\author{
SATOKO. SATOH , M. INOUE, K.M. SHIBATA, S. KAMENO AND \\ V. MIGENES \\ National Astronomical Observatory \\ 2-21-1 Osawa, Mitaka, Tokyo, 181 JAPAN \\ N. NAKAI \\ Nobeyama Radio Observatory \\ Minamimaki, Minamisaku, Nagano 384-13 JAPAN \\ AND \\ P.J. DIAMOND \\ National Radio Astronomical Observatory, NM \\ PO Box O Socorro, NM 87801, USA
}

\section{Introduction}

NGC 3079 has very luminous water megamaser from the nucleus, the peak of the spectrum being blueshifted by $180 \mathrm{~km} \mathrm{~s}^{-1}$ from the systemic velocity of the galaxy $\left(V_{\mathrm{sys}}=1131 \mathrm{~km} \mathrm{~s}^{-1}\right)$ (Henkel et al. 1984, Haschick \& Baan 1985). Core-jet like continuum structure is also found in the nuclear region (Irwin \& Seaquist 1988). No velocity drift for main features of water maser $\left(V_{L S R}=941-975 \mathrm{~km} \mathrm{~s}^{-1}\right)$ has been shown (Nakai et al. 1995). However, the drift was recently detected for the maser of $1190 \mathrm{~km} \mathrm{~s}^{-1}$ (Nakai 1997). $\mathrm{HI}$ and $\mathrm{OH}$ absorptions are detected in the nucleus (Haschick \& Baan 1985, Irwin \& Seaquist 1991). Thus this galaxy is very unique object to investigate water masers, continuum structure and absorption features all together with VLBI.

\section{Observational Results}

We conducted multi-frequency observations towards the nucleus of NGC 3079 using both Japan VLBI Network and the VLBA with phased VLA and Effelsberg. We detected two continuum components (A and B) which was consistent with the $5-\mathrm{GHz}$ result (Irwin and Seaquist 1988) at 1.4 and 
8.4 GHz, while only one component (B) was detected at 15 and $22 \mathrm{GHz}$. Water masers around $960 \mathrm{~km} \mathrm{~s}^{-1}$, are elongated 5.5 mas in N-S direction (P.A. $-20^{\circ}$ ) along the galactic plane and are apart 6.7 mas west of $B$. The position vs. velocity diagram of water masers along the major elongation presents no clear evidence of Keplerian rotation disk in this nuclear region. Three HI absorptions were detected towards the continuum components at the velocity of 1015,1127 and $1230 \mathrm{~km} \mathrm{~s}^{-1}$. The strong absorptions $(\tau>0.5)$ are clearly separated on A for $1015 \mathrm{~km} \mathrm{~s}^{-1}$, and on B for 1230 $\mathrm{km} \mathrm{s}^{-1}$, with near $V_{\text {sys }}$ component of $1127 \mathrm{~km} \mathrm{~s}^{-1}$ on both A and B. It should be noticed that this few-pc scale velocity gradient is opposite to the 100-pc scale gradient in HI (Pedlar et al. 1996).

\section{Discussion}

If we take the velocity drift as the result of the centrifugal acceleration seen in NGC4258 (Miyoshi et al. 1995), this component should be close to the near edge of rotation disk. Hence, water masers at $940-1030 \mathrm{~km} \mathrm{~s}^{-1}$ can be considered as terminal velocity features. Furthermore, when the continuum component $B$ represents the real core and the dynamic center of the system, we can draw a rotating torus with axis through B and the maser of 1190 $\mathrm{km} \mathrm{s}^{-1}$. This explains relative velocity differences among the maser and absorption features by rotation. The absorptions at 1015 and $1230 \mathrm{~km} \mathrm{~s}^{-1}$ come from the torus, on the other hand, the $1127 \mathrm{~km} \mathrm{~s}^{-1}$ feature, which is seen both on A and B, may come from the outer region. The distance between the terminal masers and the rotation axis is $0.36 \mathrm{pc}$, so assuming these masers are rotating at radius of $0.36 \mathrm{pc}$ and at rotation velocity of $250 \mathrm{~km} \mathrm{~s}^{-1}$, the central mass is estimated to be $\sim 7 \times 10^{6} M_{\odot}$. The system has different rotating axis from those of the galaxy, as seen in NGC 4258 (Miyoshi et al. 1995), and systemic velocity also.

\section{References}

Haschick, A. D., \& Baan, W. A. 1985. Nature, 314, 144

Henkel, C., et al. 1984. $A \& A p, 141, \mathrm{~L} 1$

Irwin, J. A., \& Seaquist, E. B. 1988. $A p J, \mathbf{3 3 5}, 658$

Irwin, J. A., \& Seaquist, E. B. 1991. $A p J, 371,111$

Miyoshi, M., et al. 1995. Nature, 373, 127

Nakai, N. et al. 1995. PASJ, 47, 771

Nakai, N. 1997 in IAU Symposium 184.

Pedlar, A., et al. 1996. VA, 40, 91.

Trotter, A. et al. 1997. in IAU Colloquium 164. 